\title{
Research on Dynamics and Finite Element Simulation Analysis of Variable-lead Spiral Pair Substructure
}

\author{
Fei He, Xie Xu and Ke Yang \\ College of Mechanical Engineering, Nanjing University of Science and Technology, Jiangsu Nanjing 210094 China
}

\begin{abstract}
The variable-lead spiral pair mechanism is applied to the subway gate, which can improve the stability of the mechanical structure operation. This paper analyzes the variable lead spiral substructure. By the dynamics software, the motion characteristics of the lead spiral substructure is analyzed, and the finite element software is used to analyze the structural stress, contact stress and modal analysis. Through analysis, the rationality of the design of the variable lead spiral substructure is verified. The application of the variable lead spiral pair to the subway door system is very feasible.
\end{abstract}

Keywords-variable-lead spiral pair; motion characteristics; contact stress; modal analysis

\section{INTRODUCTION}

The variable-lead spiral pair substructure is applied to the subway gate, and its structure has zero self-locking property, high stability, and maintainability. As a transmission system component of the subway gate, the variable-lead spiral pair substructure can change the speed of the door opening or closing by the change of the spiral lead. The lead screw has zero lead at both ends, it can achieve the self-locking function. Meanwhile, it and simplifies the structure of the subway gate transmission system by uninstall the additional locking structure. By this structure, the reliability of the subway gate is improved, and the production cost is reduced.

The variable-lead spiral drive has high research value, and has the characteristics of simple structure, stable transmission, high precision, high efficiency and large transmission ratio. At present, the research mainly focuses on the analysis of the motion characteristics of the screw transmission mechanism, the fitting of the transition curve at the lead change, the mechanism analysis of the variable lead screw transmission, and the machining simulation of the variable lead screw, and so on. Gallina P [1] and others improve the stability of the screw running, proposed a method to increase the moment of inertia and eliminate self-excited vibration. Chen Yong [2] and others have established a mathematical model of ball screw stiffness to analyze the factors affecting the ball screw pair; $\mathrm{Xu} \mathrm{ZZ}$ [3] designed a high-precision, high-speed ball screw pair for cooling air, its structure can reduce thermal error and improve positioning accuracy. Shen-Tarng Chiou et al. [4] studied the motion characteristics of a variable lead screw nut mechanism for high-speed shuttleless looms and studied the effects of different input powers on torque. Ming J.T [5] et al. compared the variable lead screw mechanism by point contact hypothesis and spiral theory, and used the comparison results to optimize the relevant motion characteristics and improve the transmission performance of the mechanism. HS Yan et al.
[6] studied the envelope surface of the variable lead screw and studied the meshing characteristics of the involute and helicoid of various shape surfaces. Wei Zhang [7] applied the Hertz theory to calculate the stress, and finally calculated the load spectrum through the relevant software to obtain the structural contact fatigue.

Since the variable lead helical substructure is shifted according to the lead change, the requirements of the spiral pair structure is high. Therefore, it is necessary to study the rationality of the design of the variable lead screw mechanism, and analyze the feasibility of its application to the subway gate to ensure the operation of the subway gate. In order to verify the rationality of the design of the variable-lead spiral pair substructure, this paper research the structure of the variable-lead spiral pair, and uses the virtual prototyping software ADAMS to analyze the motion of the variable-lead spiral pair substructure and calculate the corresponding speed curve. The finite element software ABAQUS performs contact stress analysis and structural modal analysis to determine the feasibility of the variable-lead spiral pair applied to the subway gate system.

\section{DYNAMIC ANALYSIS OF VARIABLE-LEAD SPIRAL PAIR SUBSTRUCTURE}

The structure of the variable-lead spiral pair is shown in Figure I. Among them, 1 is a variable lead screw, 2 is a nut, 3 is a pin roller, 4 is a spiral groove. The variable lead spiral pair is a linear motion that is converted into a nut by the rotary motion of the lead screw.

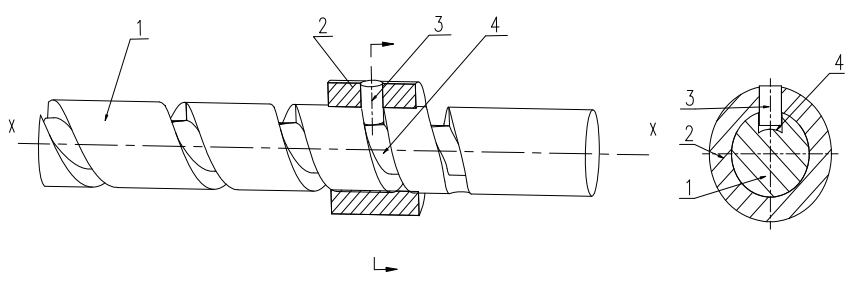

FIGURE I. SCHEMATIC DIAGRAM OF THE STRUCTURE OF THE VARIABLE-LEAD SPIRAL PAIR

In order to confirm the motion of the variable-lead spiral pair can meet the speed requirement and conforms to the motion law, the ADAMS is utilized to do the dynamic analysis. The movement mode of the variable lead screw pair is that the screw rotates to drive the linear motion of the nut, and a rotary drive is applied to the screw. The roller acts as a transmitting force, and a force is applied to the nut. By add the constraints, driving and stress conditions of the variable-lead 
spiral pair substructure in the model of the ADAMS, the analysis results are shown as Figure II.

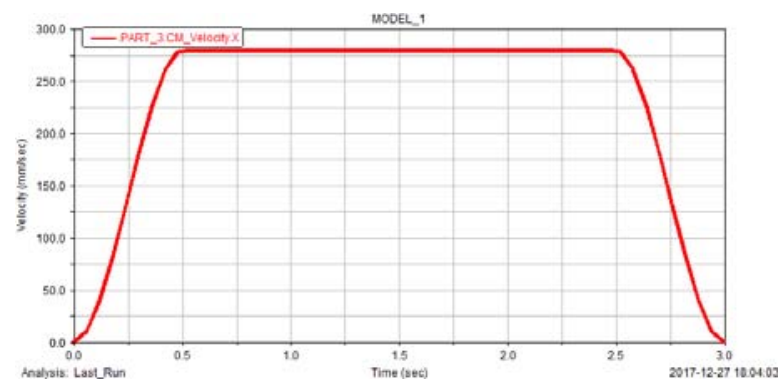

FIGURE II. SPIRAL PAIR MOVEMENT SPEED

According to the spiral sub-motion speed curve of Figure II, the motion acceleration of the variable-lead spiral pair in the starting and stopping phases is approximately constant. It indicating that the entire shifting process of the helical pair is relatively stable, and the intermediate running phase is uniform motion. Through the whole start-stop process, the movement process of the subway gate from uniform acceleration-constant-even deceleration is realized, and the variable-speed movement according to the lead change is realized, and the operation is stable. By ADAMS analysis of the motion characteristics of the variable-lead spiral pair substructure, it can be considered that the motion of the variable-lead spiral pair conforms to the motion law, meets the axial velocity requirements, and verifies the rationality of its structural design.

\section{CONTACT STRESS ANALYSIS OF VARIABLE-LEAD SPIRAL PAIR SUBSTRUCTURE}

The variable-lead spiral pair is mainly composed of three parts: a variable lead screw, a roller and a nut. Through the analysis of the spiral lead structure of the variable lead, it can be found that the whole structure is mainly concentrated on the lead screw and the roller. In order to judge the life of the roller, the contact force analysis of the roller and the screw should be performed. Here we use ABAQUS finite element simulation software to analysis the contact force of roller and the screw.

Applying a fixed constraint to both ends of the lead screw, limiting its six degrees of freedom; applying a force on the roller and limiting its five degrees of freedom, applying force at this reference point effect. The contact between the roller and the screw is established, the surface of the screw is the main contact surface, and the surface of the roller is the contact surface.

Taking the calculation result of the force of the subway gate as an example, the contact force of $127 \mathrm{~N}$ is loaded between the roller and screw for analysis. After the finite element analysis, the force and displacement cloud diagrams of the roller and screw are finally obtained, as shown in Figure III.
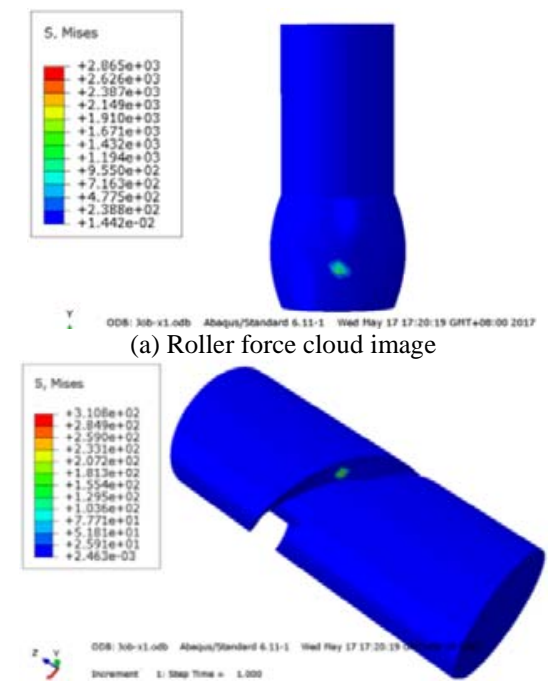

(b) Screw force cloud image

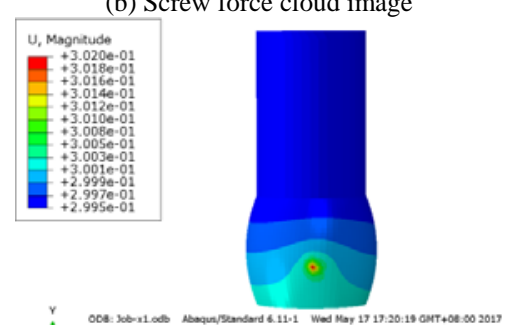

(c) Roller displacement cloud map

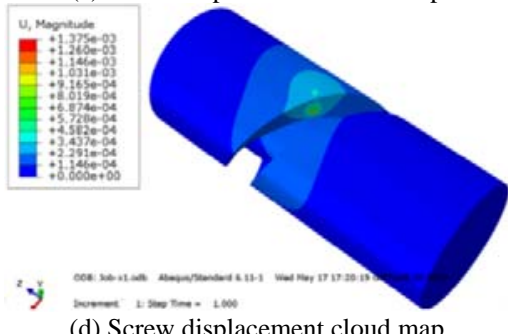

(d) Screw displacement cloud map

FIGURE III. STRESS CLOUD DIAGRAM OF THE ROLLER AND SCREW

Through the contact stress analysis of the roller and the lead screw, it can be concluded that the maximum stresses on the lead screw and the roller are: $3.108 \mathrm{e} 2 \mathrm{MPa}$ and $2.865 \mathrm{e} 3 \mathrm{MPa}$, respectively, and the actual stress of the roller is greater than Screw.

The roller makes a rolling motion in the screw. Each time the reciprocating screw is fixed, the fixing point is only subjected to two stresses. But the roller is different, the force is much more than twice; and the spiral substructure has only one roller. The rollers connect the lead screw and the nut and transmit motion and force. Compared with the ordinary ball screw, the roller has more concentrated force and higher requirements on the structure of the roller. It is necessary to analyze the life of the roller to verify the reliability of the variable-lead spiral pair substructure. 


\section{Modal ANAlysis of VARIABLE-LEAd SPIRAL PAIR SUBSTRUCTURE}

Modal analysis is a method to study the dynamic characteristics of structures. Modality refers to the natural vibration characteristics of mechanical structures. Each mode has a specific natural frequency, damping ratio and mode shape. The modal analysis is used to find out the weak links of the structure. In the design, external vibration is avoided to affect the structure, and the resonance phenomenon is avoided to affect the service life of the structure.

The first six modes are analyzed here. Fix the ends of the variable lead screw in the software, set the material properties and mesh. The final result is shown in Figure IV.

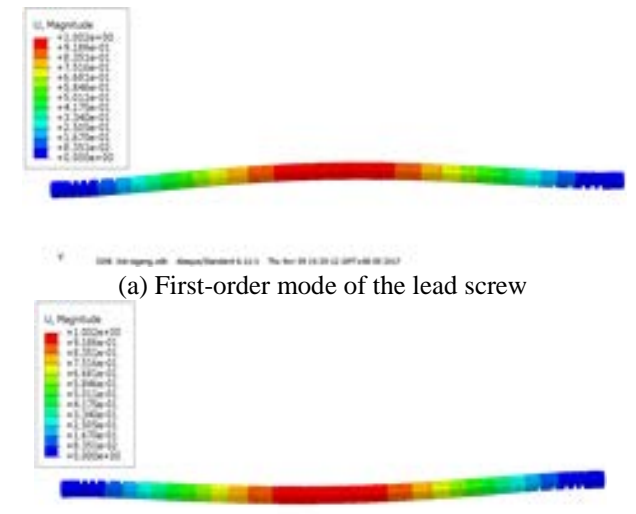

(b) Second-order mode of the lead screw

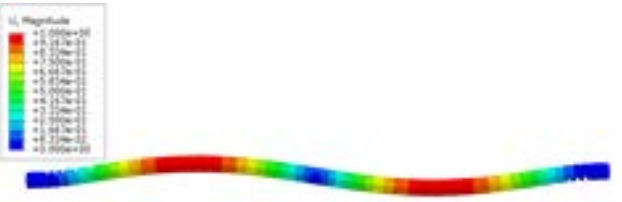

(c) Third-order mode of the lead screw

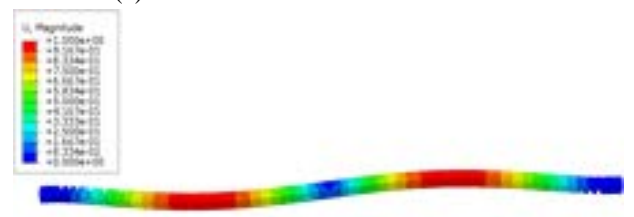

(d) Fourth-order mode of the lead screw

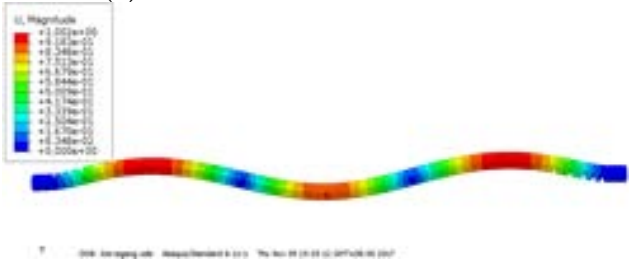

(e) Fifth-order mode of the lead screw

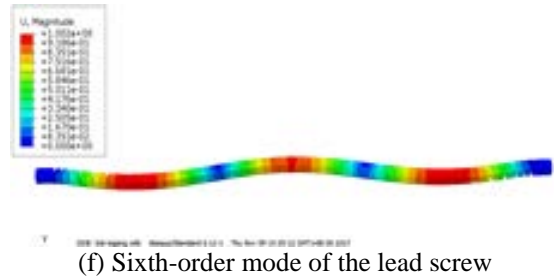

FIGURE IV. SIXTH-ORDER MODE SHAPE OF THE LEAD SCREW

The first six modal frequency values are shown in Table I.

TABLE I. THE FIRST SIX MODAL FREQUENCY VALUES

\begin{tabular}{|c|c|c|c|}
\hline Order & Frequency $(\mathrm{Hz})$ & Order & Frequency $(\mathrm{Hz})$ \\
\hline 1 & 117.98 & 4 & 357.23 \\
\hline 2 & 118.56 & 5 & 716.94 \\
\hline 3 & 355.87 & 6 & 720.00 \\
\hline
\end{tabular}

According to Figure VII, in the first six-order mode shape of the lead screw, the first-order and second-order modes are the bending of the lead screw, the first order is upward, the second order is downward, and the direction is opposite. The third-order and fourth-order modes are the torsion of the variable lead screw, and the third-order and fourth-order directions are opposite. The fifth-order and sixth-order are combinations of the lead screw and the torsion of the variable lead. It can be seen from Table 1 that the lowest natural frequency of the variable lead screw is $117.98 \mathrm{~Hz}$. According to the data, the frequency of the subway running on the ground is $30-80 \mathrm{~Hz}$, and the lowest frequency of the lead screw exceeds this range. It is judged that the variable lead screw does not generate resonance phenomenon, and the rationality of the variable-lead spiral pair substructure is determined.

\section{SUMMARY}

In this paper, the dynamics and finite element simulation analysis of the variable-lead spiral pair substructure are mainly studied. The dynamic analysis software ADAMS is used to analyze the actual movement speed of the variable-lead spiral pair, and the rationality of the variable-lead spiral pair substructure is verified. Then the finite element software ABAQUS is used to analyze the stress of the spiral roller in the spiral groove. The analysis results determine the force and dangerous position of the structure, the first six modes of the structure are also analyzed to verify the safety of the design of the spiral lead structure of the variable lead, which provides a basis for the life analysis of the variable-lead spiral pair.

In summary, the results show that the design of the variable-lead spiral pair substructure is reasonable. The running speed of the variable-lead spiral pair meets the axial speed requirement. In the spiral substructure, the roller is subjected to large stress, and the variable-lead spiral pair is rolled. The structure of the sub-structure has higher requirements. In order to improve the service life of the spiral sub-structure of the whole variable lead, it is necessary to further analyze the fatigue life of the roller and improve the feasibility of applying the variable-lead spiral pair to the subway gate system.

\section{ACKNOWLEDGEMENT}

This research was financially supported by Jiangsu Natural 
Science Foundation (BK20151481).

\section{REFERENCES}

[1] Gallina P. Vibration in screw jack mechanisms: experimental results[J]. Journal of Sound \& Vibration, 2005, 282(3-5):1025-1041.

[2] Chen Yongjiang, Tang Wencheng, Wang Jiexi. Influencing Factors and Experimental Research of Ball Screw Pair Stiffness[J]. Journal of Vibration and Shock, 2013, 32(11): 70-74.

[3] Xu Z Z, Liu X J, Choi C H, et al. A novel high speed/high precision ball screw[J]. International Journal of Precision Engineering and Manufacturing, 2013, 14(1):165-167.

[4] S.-T. C, Chen F Y. Kinetostatic analysis of variable lead screw mechanisms with $3^{\circ}$ conic frustum meshing elements[J]. Mathematical \& Computer Modelling, 1998, 27(1):17-30.

[5] Ming J T, Jan-Shiung S, Jan-Chung C. Kinematic design optimization of the variable lead screw mechanism with cone meshing element[J]. Mechanism \& Machine Theory, 1996, 31(8):1081-1093.

[6] Yan H S, Cheng H Y. The generation of variable pitch lead screws by profiles of pencil grinding wheels[J]. Mathematical \& Computer Modelling, 1997, 25(3):91-101.

[7] Zhang W, Shi X, Li D. Research on Contact Fatigue of Variable Lead Screw System[J]. International Journal of Digital Content Technology \& Its Applications, 2013, 7(10):1-9 